\title{
Combined neutrophil-platelet score and hemoglobin level predict survival in esophageal squamous cell carcinoma patients treated with chemoradiotherapy
}

\author{
Chuanwang Miao ${ }^{1,2, *}$, Shan Zhu ${ }^{3,4, *}$, Hong Pan ${ }^{5}$, Xiaolan Cao ${ }^{1,2}$, Shuanghu Yuan² \\ and Xudong $\mathrm{Hu}^{2}$ \\ ${ }^{1}$ School of Medicine and Life Sciences, University of Jinan-Shandong Academy of Medical Sciences, Jinan, Shandong Province, \\ P.R. China \\ ${ }^{2}$ Department of Radiotherapy, Shandong Cancer Hospital Affiliated to Shandong University, Jinan, Shandong Province, \\ P.R. China \\ ${ }^{3}$ Department of Head and Neck Radiotherapy, Shandong Provincial Hospital Affiliated to Shandong University, Jinan, \\ Shandong Province, P.R. China \\ ${ }^{4}$ Department of Head and Neck Radiotherapy, Shandong Provincial Western Hospital, Jinan, Shandong Province, P.R. China \\ ${ }^{5}$ Central Sterile Supply Department, Shandong Cancer Hospital Affiliated to Shandong University, Jinan, Shandong Province, \\ P.R. China \\ *These authors have contributed equally to this work
}

Correspondence to: Xudong Hu, email: drhuxudong@163.com

Keywords: chemoradiotherapy (CRT), esophageal squamous cell carcinoma (ESCC), neutrophil-to-lymphocyte ratio (NLR), platelet-to-lymphocyte ratio (PLR), hemoglobin ( $\mathrm{Hb})$

Received: June 21,2017 Accepted: August 17, $2017 \quad$ Published: September 27, 2017

Copyright: Miao et al. This is an open-access article distributed under the terms of the Creative Commons Attribution License 3.0 (CC BY 3.0), which permits unrestricted use, distribution, and reproduction in any medium, provided the original author and source are credited.

\section{ABSTRACT}

Systemic inflammation and hematological markers have prognostic value in patients with esophageal squamous cell carcinoma (ESCC). The objective of this study was to evaluate the neutrophil-to-lymphocyte ratio (NLR), platelet-to-lymphocyte ratio (PLR), combined neutrophil-platelet (CNP) score, and hemoglobin (Hb) to inform treatment decisions and predict outcomes in patients with locally advanced ESCC treated with chemoradiotherapy (CRT). A total of 168 patients with locally advanced ESCC were retrospectively evaluated. Patients were stratified by marker value using a receiver operating characteristic curve analysis to determine the cutoff point. Logistic regression was used to identify markers associated with sensitivity to treatment. Overall survival (OS) was calculated by the Kaplan-Meier method. Multivariate Cox logistic regression modeling was used to assess the influences of OS. Smoking history, tumour site, clinical stage, NLR, PLR, CNP, and Hb ( $p \leq 0.05)$ were associated with the sensitivity to therapy. In multivariate analysis, a high CNP score was independently associated with poor treatment sensitivity (OR $=2.066, p$ $=0.021$ ). Univariate analysis revealed that PLR, CNP, and Hb levels were associated with OS, and Cox multivariate analysis found that CNP $(H R=1.47, p=0.027)$ and Hb level $(H R=0.44, p=0.007)$ were independent predictors of OS. In conclusion, CNP and $\mathrm{Hb}$ are inexpensive and universally available prognostic markers in patients with locally advanced ESCC patients. CNP score is a systemic inflammatory marker that predicted sensitivity to CRT. 


\section{INTRODUCTION}

Esophageal cancer is prevalent in China [1], with an estimated incidence of 21.62/100,000 and estimated mortality of $16.25 / 100,000$ population in 2011 [1]. Esophageal squamous cell carcinoma (ESCC) is the most common type of cancer, accounting for $88.8 \%$ of all diagnosed cases [1]. The incidence of ESCC has decreased over the past 20 years, but the prognosis remains very poor, with an overall 5 -year survival rate of approximately $20 \%$ and only $3.8 \%$ for advanced disease [2]. Improved diagnostic and prognostic markers and individualized treatment strategies are needed to improve prognosis.

The tumor-associated inflammatory response may reflect either compromised host immune function or an antitumor immune response [3]. Systemic inflammation can be evaluated with routinely available hematological and clinical laboratory testing and is of interest as a potential marker of treatment response [3]. Studies of ESCC pathogenesis and advances in precision and personalized medicine for ESCC patients have increased efforts to identify inflammatory markers and clinicopathological characteristics with prognostic value [4]. Recent evidence has supported systemic tumorassociated inflammatory response markers, including $\mathrm{C}$-reactive protein (CRP) and the Glasgow prognosis score (GPS) as able to predict the outcome in ESCC patients treated with surgery $[5,6]$. Similar studies have not been conducted in ESCC patients treated with chemoradiotherapy (CRT), and CRP is not routinely evaluated in those patients.

Correlation of presurgical neutrophil-tolymphocyte ratio (NLR) and platelet-to-lymphocyte ratio (PLR) and poor prognosis have been reported in patients with lung, liver, breast, and colorectal cancer [7-10]. Hyder et al. reported changes in the NLR and PLR during CRT that predicted survival and pathologic complete response in ESCC patients [11], but relationships of inflammatory factors and CRT sensitivity were not reported. Patients with ESCC experience physical decline because of malnutrition resulting from impaired ability to swallow and systemic effects of malignancy. A reduction in hemoglobin ( $\mathrm{Hb})$ in such patients has prognostic value following surgery or CRT [12-14].

Evidence of systemic inflammatory and hematological variables as markers of CRT response and outcome in patients with locally advanced ESCC is limited. We previously demonstrated that NLR, CNP, and clinical stage were significantly associated with CRT sensitivity in patients with ESCC [15]. The objective of this study was to investigate the value of NLR, PLR, $\mathrm{CNP}$, and $\mathrm{Hb}$ in patients with locally advanced ESCC and CRT to inform treatment decisions and predict treatment outcomes.

\section{RESULTS}

\section{Patient demographics}

Of the 168 patients who were included, 134 were men $(79.8 \%), 34$ were women $(20.2 \%)$, and their average age was $67.15 \pm 8.86$ years. The patient clinicopathological characteristics (gender, age, smoking history, tumor site, tumor stage) are summarized in Table 1. The clinical responses following therapy are shown in Table 2, and patient survival is reported in Table 3.

\section{Cutoff points of potential predictive serum markers}

The optimum cutoff points of the NLR, PLR, and $\mathrm{Hb}$ level calculated by the maximum values of the Youden index were the test variables, and treatment response was the static variable that was used to construct the ROC curve. The optimal critical values for predicting the response to therapy were an NLR of 3.34, a PLR of 103.75, and an $\mathrm{Hb}$ concentration of $43.98 \mathrm{~g} / \mathrm{L}$; the area under the curve (AUC) was 0.520, 0.505, and 0.505. The ROC curve was conducted for OS as a static variable. The optimal critical values associated with improved OS were an NLR of 3.51, a PLR of 145.41, and an Hb concentration of $132.50 \mathrm{~g} / \mathrm{L}$; the AUC was $0.501,0.503$, and 0.607 .

\section{Treatment response and predictive markers}

Of the 168 included patients, $124(73.8 \%)$ had a complete response (CR) or a partial response (PR); 44 patients $(26.2 \%)$ had stable disease (SD) or progressive disease (PD). The analysis of relationships between treatment response and patient variables included age, sex, smoking history, tumor location, clinical stage, NLR, PLR, $\mathrm{CNP}$ score, and $\mathrm{Hb}$ level. Univariate analysis were found significant relationships between response and smoking history $(p<0.001)$, tumour site $(p=0.05)$, clinical stage $(p<0.001)$, NLR $(p=0.003), \operatorname{PLR}(p=0.005), \operatorname{CNP}(p<$ $0.001)$, and Hb level $(p<0.001)$ (Table 2). In subsequent logistic multifactor analysis, only a high CNP score (HR $=2.066, p=0.021)$ was independently associated with sensitivity to therapy (Table 4 ).

\section{Survival outcomes and prognostic factors}

The median OS was 33 (range 2-36) months. Threeyear OS was better in patients with low (58.3\%) than with high (30.6\%) PLRs or low (59.5\%) than with high $(25.0 \%)$ CNP scores (both $p<0.05$; Figure 1A, 1B). Three-year OS was better in patients with high $(56.4 \%)$ than with low (27.6\%) Hb level ( $p<0.05$; Figure 1C). Kaplan-Meier survival curves (Figure 1D) show that 3-year OS of patients with low NLRs $(54.5 \%)$ was better than that of those with high NLRs (31.0\%), but the difference was not significant. 
Table 1: Patient group and demographic characteristics

\begin{tabular}{lc}
\hline Factor & Patient $(\mathbf{n = 1 6 8 )}$ \\
\hline Age $(\leq 60 />60)$ & $32(19.0 \%) / 136(81.0 \%)$ \\
Sex (male/female) & $134(79.8 \%) / 34(20.2 \%)$ \\
Smoking (no/yes) & $72(42.9 \%) / 96(57.1 \%)$ \\
Tumour site (Upper 1/3/Middle 1/3/Lower $1 / 3)$ & $60(35.7 \%) / 78(46.4 \%) / 30(17.9 \%)$ \\
T stage (II/III/IV) & $24(14.3 \%) / 106(63.1 \%) / 38(22.6 \%)$ \\
N stage (0/I) & $44(26.2 \%) / 124(73.8 \%)$ \\
Clinic stage (II/III/IV) & $44(26.2 \%) / 86(51.2 \%) / 38(22.6 \%)$ \\
Adjuvant therapies (RT/CCRT) & $46(27.4 \%) / 122(72.6 \%)$ \\
\hline
\end{tabular}

RT: radical radiotherapy only; CCRT: concurrent chemoradiotherapy.

Univariate analysis of the relationship of age, sex, smoking history, tumor location, clinical stage, NLR, PLR, CNP score, and $\mathrm{Hb}$ level and prognosis revealed that elevated PLR ( $p=0.019)$, elevated CNP score $(p=0.048)$, and a decreased $\mathrm{Hb}$ level $(p=0.003)$ were associated with decreased OS. Multivariate analysis showed that elevated CNP score $(\mathrm{HR}=1.465, p=0.027)$, and decreased $\mathrm{Hb}$ level $(\mathrm{HR}=0.444, p=0.007)$ were both independent prognostic markers of decreased OS (Table 5).

\section{DISCUSSION}

This study is one of only a few to evaluate the prognostic value of hematological markers of systemic inflammation and nutritional state for clinical outcome for patients with locally advanced ESCC treated with CRT. Evaluation of NLR and PLR can easily be performed routinely in clinical laboratories, and univariate analysis found that reduced NLR and PLR were associated with increased effectiveness of therapy and with improved OS. The study also found that a high CNP score was an independent risk factor affecting CRT sensitivity and patient outcome. Hb level also had prognostic value. These initial findings warrant further study in larger, controlled prospective studies of ESCC patients treated with CRT to provide evidence-based guidance for the use of these laboratory tests, which are inexpensive, highly reliable, and reproducible.

The clinical outcomes of cancer patients depend on both the characteristics of the tumor itself and on host responses [16], including inflammatory and immune responses that limit the cancer development and progression [17]. Cancer cells may secrete growth factors, including granulocyte colony-stimulating factor (G-CSF) or granulocyte macrophage colonystimulating factor (M-CSF), both of which stimulate an increase in the number of peripheral blood neutrophils
[18]. Neutrophils produce tumor-associated vascular endothelial growth factor, interleukin, and tumor necrosis factor, all of which can disrupt the tumor stroma to facilitate invasion and metastasis [19]. Cancer cells may also release inflammatory mediators that stimulate megakaryocytes in the bone marrow, which results in an increase in platelets in the peripheral blood [20]. Blood platelets can also promote the invasion and metastasis of tumor cells [21]. Lymphocytes, primarily $\mathrm{CD}^{+} \mathrm{T}$ cells and NK cells, identify and clear tumor cells [22]; reduction in the number of lymphocytes weakens the antitumor immune response and increases the probability of tumor cell immune escape and becoming lost to immune system monitoring. Increased tumor growth leads to reduced CRT sensitivity and poor prognosis. Previous studies of the effects of $\mathrm{Hb}$ level on patient prognosis in cancer $[12,13]$ indicate that increased $\mathrm{Hb}$ levels lead to reduced tumor hypoxia and improved CRT sensitivity, thus improving the therapeutic effect [14]. The measurement of Hb levels may thus be included in a prognosis scoring system for cancer ESCC patients undergoing CRT [23].

Previous studies that evaluated the association of NLR, PLR, and patient outcome in 1,540 patients with esophagectomy [24-29] reported that elevated NLR and PLR were associated with poor prognosis. However, less data are available in patients not indicated for surgery and treated by CRT. Multivariate analysis in this patient series showed that PLR and NLR alone were not independent predictors of patient outcome. Previous studies of the GPS, which includes the assay of CRP, found that it was associated with reduced survival of ESCC patients [6]. However, CRP is not routinely included in routine laboratory testing prior to CRT. In this study, CNP score was an independent predictor of patient outcome and CRT sensitivity. In the evaluation, CRT sensitivity and variables with a significant effect in univariate analysis were 
Table 2: Univariate analyses of sensitivity to chemoradiotherapy in ESCC patients

\begin{tabular}{|c|c|c|c|c|c|}
\hline Factor & $\mathbf{N}$ & Responder & Resistance & Chi-square & $p$ value \\
\hline \multicolumn{6}{|l|}{ Age } \\
\hline$\leq 60$ & 32 & 22 & 10 & 0.523 & 0.505 \\
\hline$>60$ & 136 & 102 & 34 & & \\
\hline \multicolumn{6}{|l|}{ Sex } \\
\hline Male & 134 & 100 & 34 & 0.229 & 0.665 \\
\hline Female & 34 & 24 & 10 & & \\
\hline \multicolumn{6}{|l|}{ Smoking } \\
\hline No & 72 & 42 & 30 & 15.611 & $<0.001$ \\
\hline Yes & 96 & 82 & 14 & & \\
\hline \multicolumn{6}{|l|}{ Tumour site } \\
\hline Upper $1 / 3$ & 60 & 54 & 10 & 5.979 & 0.05 \\
\hline Middle $1 / 3$ & 78 & 50 & 24 & & \\
\hline Lower $1 / 3$ & 30 & 20 & 10 & & \\
\hline \multicolumn{6}{|l|}{ T stage } \\
\hline II/III & 130 & 98 & 32 & 0.738 & 0.390 \\
\hline IV & 38 & 26 & 12 & & \\
\hline \multicolumn{6}{|l|}{$\mathrm{N}$ stage } \\
\hline 0 & 44 & 34 & 10 & 0.370 & 0.690 \\
\hline I & 124 & 90 & 34 & & \\
\hline \multicolumn{6}{|l|}{ Clinic stage } \\
\hline II & 44 & 34 & 10 & 17.811 & $<0.001$ \\
\hline III & 86 & 70 & 14 & & \\
\hline IV & 38 & 18 & 20 & & \\
\hline \multicolumn{6}{|c|}{ Adjuvant therapies } \\
\hline $\mathrm{RT}$ & 46 & 30 & 16 & 2.419 & 0.167 \\
\hline CCRT & 122 & 94 & 28 & & \\
\hline \multicolumn{6}{|l|}{ NLR } \\
\hline$\leq 3.34$ & 108 & 88 & 20 & 9.207 & 0.003 \\
\hline$>3.34$ & 60 & 36 & 24 & & \\
\hline \multicolumn{6}{|l|}{ PLR } \\
\hline$\leq 103.75$ & 68 & 58 & 10 & 7.795 & 0.005 \\
\hline$>103.75$ & 100 & 66 & 34 & & \\
\hline \multicolumn{6}{|l|}{$\mathrm{CNP}$} \\
\hline 0 score & 64 & 64 & 10 & 15.283 & $<0.001$ \\
\hline 1 score & 48 & 38 & 10 & & \\
\hline 2 score & 56 & 32 & 24 & & \\
\hline \multicolumn{6}{|c|}{ Hemoglobin level } \\
\hline$\leq 43.98$ & 80 & 50 & 30 & 10.105 & $<0.001$ \\
\hline$>43.98$ & 88 & 74 & 14 & & \\
\hline
\end{tabular}


Table 3: Univariate analyses of OS in ESCC patients

\begin{tabular}{|c|c|c|c|}
\hline & 3-year OS & $\begin{array}{c}\text { Log rank } \\
\text { (Chi-square) }\end{array}$ & $p$ value \\
\hline $\operatorname{Age}(\leq 60 />60)$ & $56.3 \% / 44.4 \%$ & 0.341 & 0.559 \\
\hline Sex( Male/Female) & $46.3 \% / 47.1 \%$ & 0.012 & 0.912 \\
\hline Smocking (No/Yes) & $41.7 \% / 50.0 \%$ & 0.456 & 0.499 \\
\hline $\begin{array}{l}\text { Tumour site } \\
\text { (Upper1/3, Middle 1/3, } \\
\text { Lower 1/3) }\end{array}$ & $43.3 \% / 51.3 \% / 40.0 \%$ & 0.315 & 0.575 \\
\hline T stage (II, III/IV) & $58.3 \% / 49.1 \% / 31.6 \%$ & 2.964 & 0.227 \\
\hline $\mathrm{N}$ stage $(0 / \mathrm{I})$ & $59.1 \% / 41.9 \%$ & 2.304 & 0.129 \\
\hline Clinic stage (II, III/IV) & $68.2 \% / 37.2 \% / 42.1 \%$ & 4.769 & 0.092 \\
\hline therapies (RT/CCRT) & $30.4 \% / 52.2 \%$ & 0.930 & 0.335 \\
\hline $\operatorname{NLR}(\leq 3.34 />3.34)$ & $60 \% / 31.0 \%$ & 2.809 & 0.094 \\
\hline $\operatorname{PLR}(\leq 103.75 />103.75)$ & $58.3 \% / 30.6 \%$ & 5.491 & 0.019 \\
\hline $\mathrm{CNP}(0 / 1 / 2$ score $)$ & $59.5 \% / 47.1 \% / 24.0 \%$ & 6.078 & 0.048 \\
\hline Hemoglobin $(\leq 43.98 />43.98)$ & $27.6 \% / 56.4 \%$ & 8.784 & 0.003 \\
\hline
\end{tabular}

Table 4: Multivariate analyses of chemoradiotherapy in ESCC patients

\begin{tabular}{lcccc}
\hline & \multirow{2}{*}{$\boldsymbol{p}$ value } & OR & \multicolumn{2}{c}{ OR 95\%CI } \\
\cline { 3 - 5 } & & & upper limit & lower limit \\
\hline CNP & 0.021 & 2.066 & 1.114 & 3.833 \\
\hline
\end{tabular}

included in a multivariate logistic regression analysis, which confirmed that a high CNP score was independently associated with sensitivity to therapy $(\mathrm{OR}=2.066, p=$ 0.021), and Cox multifactorial model analysis found that CNP score was an independently associated with improved OS $(\mathrm{OR}=1.47, p=0.027)$.

The measurement of $\mathrm{Hb}$ levels is routinely performed for all patients on admission to the hospital. Although it has low specificity, reduced $\mathrm{Hb}$ levels are often found in patients with cancer, and may indirectly reflect nutritional status. In a series of 103 ESCC patients with radiotherapy and concurrent chemotherapy, Zhang et al. found that 3-year OS was reduced in patients with anemia [23]. In this study, patients with normal or high $\mathrm{Hb}$ levels had an improved sensitivity to CRT, and the Cox multifactorial model found that $\mathrm{Hb}$ was an independent predictor of OS. Therefore, early individualized treatment and nutritional support may have both short-term efficacy and long-term survival benefit.
The study limitations include its single-center retrospective design and small patient sample size. Also, the predictive value of the inflammation-associated serological factors such as the GPS could not be evaluated because they are not performed in routine clinical examinations. Histological evaluation of the relationship between tissue and systemic neutrophilia to confirm that neutrophilia was a consequence of the tumor, rather than another factor, such as infection, could not be done. Larger, controlled prospective clinical studies are warranted to confirm the predictive value of these inflammatory markers on the response to CRT and on the long-term survival of patients with locally advanced ESCC. In conclusion, despite the acknowledged limitations, CNP score offers additional sensitivity to the choice of CRT for the clinical management of locally advanced ESCC. CNP score and $\mathrm{Hb}$ level had prognostic value in locally advanced ESCC. This analysis showed that a low CNP score was the risk factor and high $\mathrm{Hb}$ level appeared to be protective. 
Table 5: Multivariate analyses of OS in ESCC patients

\begin{tabular}{lcccc}
\hline & p value & HR & & HR 95\%CI \\
\cline { 4 - 5 } & & & upper limit & upper limit \\
\hline CNP & 0.027 & 1.465 & 1.045 & 2.052 \\
Hemoglobin & 0.007 & 0.444 & 0.245 & 2.052 \\
\hline
\end{tabular}
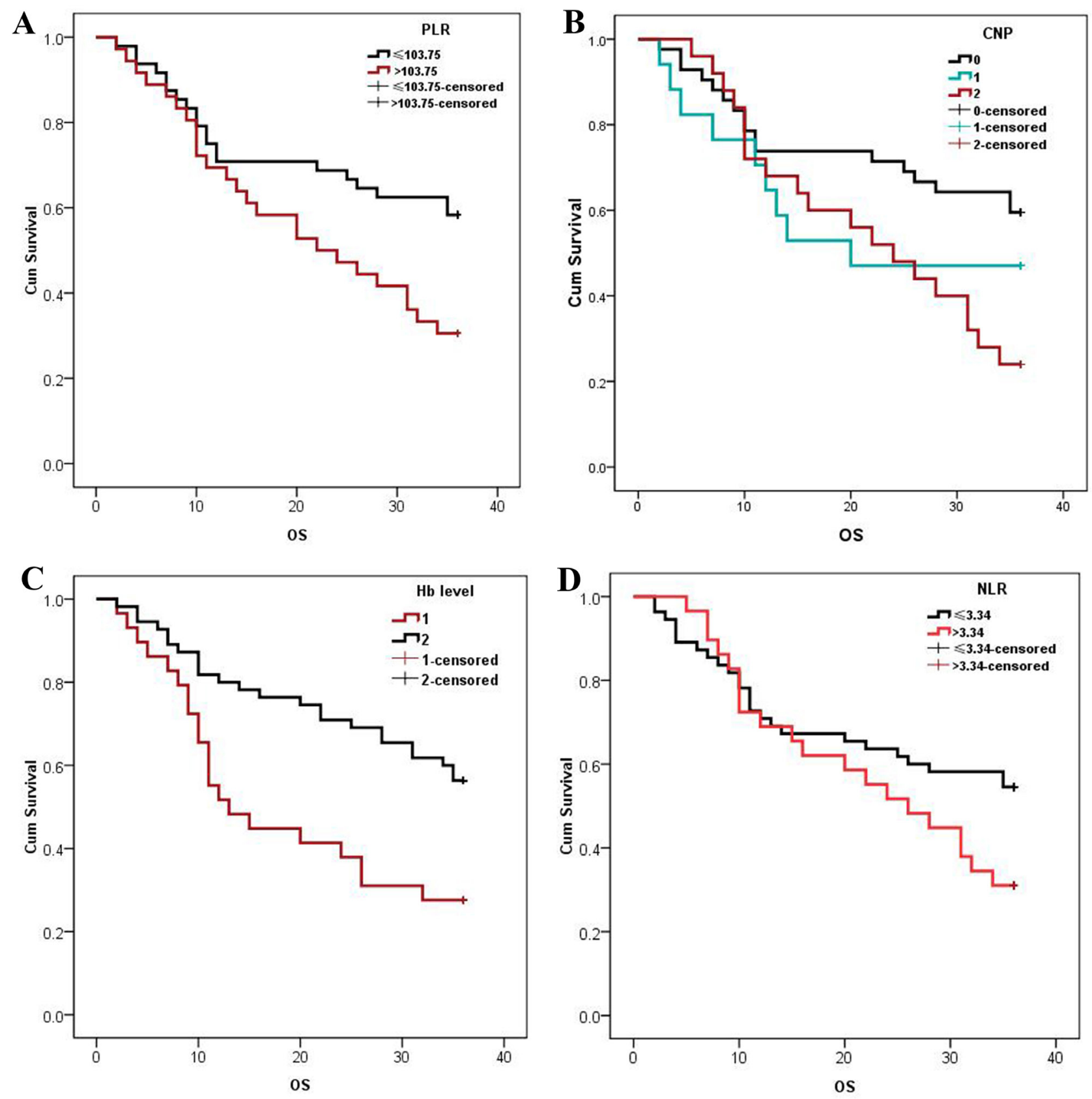

Figure 1: Kaplan-Meier estimates of OS of patients stratified by PLR (A), CNP (B), Hb level (C) and NLR (D). Patients with PLRs $>103.75$, CNP scores of 3 , and a $\mathrm{Hb}$ concentration $<43.98 \mathrm{~g} / \mathrm{L}$ had significantly decreased $\mathrm{OS}(\mathrm{P}<0.05)$. The 3 -year cumulative OS of patients with an NLR $\leq 3.34$ was $54.5 \%$ and was $31.0 \%$ in those with an NLR $>3.34$. 


\section{MATERIALS AND METHODS}

\section{Patients}

This retrospective study included patients with locally advanced ESCC with CRT at Shandong Cancer Hospital Affiliated to Shandong University between 2006 and 2012. Patients with histologically confirmed ESCC treated with CRT, available laboratory and hematological testing results within 7 days of treatment, and available clinicopathological and follow-up data were eligible. ESCC patients with acute or chronic infectious disease or disease-associated complications; targeted antitumor therapy; liver, kidney, or autoimmune disease; thrombosis or bleeding disorders were excluded. All patients reviewed the study protocol and gave written informed consent before participation. The Ethics Committee of Shandong Cancer Hospital Affiliated to Shandong University approved the study.

\section{Evaluation of inflammation markers}

Pretreatment white blood cell, neutrophil, lymphocyte, monocyte, platelet counts, and $\mathrm{Hb}$ level were included in the data analysis. The NLR was calculated by dividing the absolute neutrophil count by the absolute lymphocyte count. The PLR was calculated by dividing the absolute platelet count by the absolute lymphocyte count. Patients with reduced NLRs and PLRs were given a combined neutrophil-platelet (CNP) score of 0 . Those with a reduced NLR or PLR were given a CNP of 1, and those with normal NLRs and PLRs were given a score of 2.

\section{Treatment}

The radiotherapy protocol included a total radiation dose of up to 59.6 Gy delivered by standard fractionated radiotherapy in 34 fractions on weekdays; $2.0 \mathrm{~Gy} / \mathrm{f} \times$ $20 \mathrm{f}, 1.4 \mathrm{~Gy} / \mathrm{f} \times 14 \mathrm{f}$; over a 6 -week cycle. Concurrent chemotherapy consisted of 2 28-day cycles of daily cisplatin $\left(75-100 \mathrm{mg} / \mathrm{m}^{2} / \mathrm{dL}\right)$ and fluorouracil $(750-1000$ $\mathrm{mg} / \mathrm{m}^{2} / \mathrm{dL}$ ).

\section{Clinical evaluation and follow-up}

Treatment evaluation followed the Response Evaluation Criteria In Solid Tumors criteria [30]. Patients with a complete response (CR) or partial response (PR) following treatment were "responders." Patients exhibiting stable disease (SD) or progressive disease (PD) were classified as "resistant". Follow-up evaluations were performed every 3 months after patients were discharged from the hospital and included a medical history, physical examination, and computed tomography of the chest. Endoscopy was performed in cases of clinical evidence of recurrence or metastasis. The last follow-up visits were in February 2015. Survival period was measured from the date of admission to the date of death, or to the date of the last follow-up.

\section{Statistical analysis}

The study data analyzed as possible predictive markers were sex, age, smoking history, tumor site, tumor stage, NLR, PLR, CNP, and Hb. Optimal cutoff values were determined by the maximum potential effectiveness values of the Youden index, a summary measure of the receiver operating characteristic (ROC) curve [31]. The chi-square test was used to analyze the relationship between possible predictive markers and clinical responses following therapy. Variables with $p$ values $\leq 0.05$ by univariate analysis were included in multivariate logistic regression analysis. Overall survival (OS) was calculated by the Kaplan-Meier method, and the significance of differences was determined by the logrank test. Multivariate Cox logistic regression modeling was used to assess the influence of predictive markers on patient survival outcomes. Statistical significance was defined as $p \leq 0.05$ or a $95 \%$ confidence interval that did not exceed 1.00. All analyses were conducted using SPSS 17.0 software.

\section{CONFLICTS OF INTEREST}

The authors declare no conflicts of interest concerning this article.

\section{FUNDING}

This study was funded by the Natural Science Foundation of China (NSFC81472812). No other potential conflicts of interest relevant to this article are reported.

\section{REFERENCES}

1. Zeng H, Zheng R, Zhang S, Zuo T, Xia C, Zou X, Chen W. Esophageal cancer statistics in China, 2011: Estimates based on 177 cancer registries. Thorac Cancer. 2016; 7:232-7.

2. Zeng H, Zheng R, Guo Y, Zhang S, Zou X, Wang N, Zhang L, Tang J, Chen J, Wei K, Huang S, Wang J, Yu L, et al. Cancer survival in China, 2003-2005: a population-based study. Int J Cancer. 2015; 136:1921-30.

3. Grivennikov SI, Greten FR, Karin M. Immunity, inflammation, and cancer. Cell. 2010; 140:883-99.

4. Noble F, Hopkins J, Curtis N, Kelly JJ, Bailey IS, Byrne JP, Bateman AC, Bateman AR, Underwood TJ. The role of systemic inflammatory and nutritional blood-borne markers in predicting response to neoadjuvant chemotherapy and survival in oesophagogastric cancer. Med Oncol. 2013; 30:596.

5. Wang CY, Hsieh MJ, Chiu YC, Li SH, Huang HW, Fang FM, Huang YJ. Higher serum C-reactive protein 
concentration and hypoalbuminemia are poor prognostic indicators in patients with esophageal cancer undergoing radiotherapy. Radiother Oncol. 2009; 92:270-5.

6. Liu JS, Huang Y, Yang X, Feng JF. A nomogram to predict prognostic values of various inflammatory biomarkers in patients with esophageal squamous cell carcinoma. Am J Cancer Res. 2015; 5:2180-9.

7. Ilie M, Hofman V, Ortholan C, Bonnetaud C, Coëlle C, Mouroux J, Hofman P. Predictive clinical outcome of the intratumoral CD66b-positive neutrophil-to-CD8-positive T-cell ratio in patients with resectable nonsmall cell lung cancer. Cancer. 2012; 118:1726-37.

8. Pinato DJ, Stebbing J, Ishizuka M, Khan SA, Wasan HS, North BV, Kubota K, Sharma R. A novel and validated prognostic index in hepatocellular carcinoma: the inflammation based index (IBI). J Hepatol. 2012; 57:1013-20.

9. Azab B, Bhatt VR, Phookan J, Murukutla S, Kohn N, Terjanian T, Widmann WD. Usefulness of the neutrophilto-lymphocyte ratio in predicting short- and long-term mortality in breast cancer patients. Ann Surg Oncol. 2012; 19:217-24.

10. Li MX, Liu XM, Zhang XF, Zhang JF, Wang WL, Zhu Y, Dong J, Cheng JW, Liu ZW, Ma L, Lv Y. Prognostic role of neutrophil-to-lymphocyte ratio in colorectal cancer: a systematic review and meta-analysis. Int J Cancer. 2014; 134:2403-13.

11. Hyder J, Boggs DH, Hanna A, Suntharalingam M, Chuong MD. Changes in neutrophil-to-lymphocyte and plateletto-lymphocyte ratios during chemoradiation predict for survival and pathologic complete response in trimodality esophageal cancer patients. J Gastrointest Oncol. 2016; 7:189-95.

12. Wang Y, Wang L, Yang Q, Li J, Qi Z, He M, Yao J, Qiao $\mathrm{X}$. Factors on prognosis in patients of stage pT3N0M0 thoracic esophageal squamous cell carcinoma after two-field esophagectomy. J Cancer Res Ther. 2015; 11:C16-23.

13. Hamai Y, Hihara J, Taomoto J, Yamakita I, Ibuki Y, Okada M. Hemoglobin level influences tumor response and survival after neoadjuvant chemoradiotherapy for esophageal squamous cell carcinoma. World J Surg. 2014; 38:2046-51.

14. Zhang F, Cheng F, Cao L, Wang S, Zhou W, Ma W. A retrospective study: the prevalence and prognostic value of anemia in patients undergoing radiotherapy for esophageal squamous cell carcinoma. World J Surg Oncol. 2014; 12:244.

15. Zhu S, Miao CW, Wang ZT, Peng L, Li B. Sensitivity value of hematological markers in patients receiving chemoradiotherapy for esophageal squamous cell carcinoma. Onco Targets Ther. 2016; 9:6187-93.

16. Hanahan D, Weinberg RA. Hallmarks of cancer: the next generation. Cell. 2011; 144:646-74.
17. Aggarwal BB, Gehlot P. Inflammation and cancer: how friendly is the relationship for cancer patients. Curr Opin Pharmacol. 2009; 9:351-69.

18. Nowarski R, Gagliani N, Huber S, Flavell RA. Innate immune cells in inflammation and cancer. Cancer Immunol Res. 2013; 1:77-84.

19. Kusumanto YH, Dam WA, Hospers GA, Meijer C, Mulder NH. Platelets and granulocytes, in particular the neutrophils, form important compartments for circulating vascular endothelial growth factor. Angiogenesis. 2003; 6:283-7.

20. Alexandrakis MG, Passam FH, Moschandrea IA, Christophoridou AV, Pappa CA, Coulocheri SA, Kyriakou DS. Levels of serum cytokines and acute phase proteins in patients with essential and cancer-related thrombocytosis. Am J Clin Oncol. 2003; 26:135-40.

21. Bambace NM, Holmes CE. The platelet contribution to cancer progression. J Thromb Haemost. 2011; 9:237-49.

22. Titu LV, Monson JR, Greenman J. The role of CD8(+) T cells in immune responses to colorectal cancer. Cancer Immunol Immunother. 2002; 51:235-47.

23. Zhang M, Zhou S, Zhang L, Ye W, Wen Q, Wang J. Role of cancer-related inflammation in esophageal cancer. Crit Rev Eukaryot Gene Expr. 2013; 23:27-35.

24. Feng JF, Huang Y, Chen QX. Preoperative platelet lymphocyte ratio (PLR) is superior to neutrophil lymphocyte ratio (NLR) as a predictive factor in patients with esophageal squamous cell carcinoma. World J Surg Oncol. 2014; 12:58.

25. Roxburgh CS, Wallace AM, Guthrie GK, Horgan PG, McMillan DC. Comparison of the prognostic value of tumour- and patient-related factors in patients undergoing potentially curative surgery for colon cancer. Colorectal Dis. 2010; 12:987-94.

26. Feng JF, Huang Y, Liu JS. Combination of neutrophil lymphocyte ratio and platelet lymphocyte ratio is a useful predictor of postoperative survival in patients with esophageal squamous cell carcinoma. Onco Targets Ther. 2013; 6:1605-12.

27. Miyata H, Yamasaki M, Kurokawa Y, Takiguchi S, Nakajima K, Fujiwara Y, Mori M, Doki Y. Prognostic value of an inflammation-based score in patients undergoing preoperative chemotherapy followed by surgery for esophageal cancer. Exp Ther Med. 2011; 2:879-85.

28. Sharaiha RZ, Halazun KJ, Mirza F, Port JL, Lee PC, Neugut AI, Altorki NK, Abrams JA. Elevated preoperative neutrophil:lymphocyte ratio as a predictor of postoperative disease recurrence in esophageal cancer. Ann Surg Oncol. 2011; 18:3362-9.

29. Xie X, Luo KJ, Hu Y, Wang JY, Chen J. Prognostic value of preoperative platelet-lymphocyte and neutrophillymphocyte ratio in patients undergoing surgery for esophageal squamous cell cancer. Dis Esophagus. 2016; 29:79-85. 
30. Eisenhauer EA, Therasse P, Bogaerts J, Schwartz LH, Sargent D, Ford R, Dancey J, Arbuck S, Gwyther S, Mooney M, Rubinstein L, Shankar L, Dodd L, et al. New response evaluation criteria in solid tumours: revised RECIST guideline (version 1.1). Eur J Cancer. 2009; 45:228-47.
31. Ruopp MD, Perkins NJ, Whitcomb BW, Schisterman EF. Youden Index and optimal cut-point estimated from observations affected by a lower limit of detection. Biom J. 2008; 50:419-30. 